Published in final edited form as:

Int J STD AIDS. 2017 August ; 28(9): 849-857. doi:10.1177/0956462416675095.

\title{
Willingness to use pre-exposure prophylaxis among Black and White men who have sex with men in Atlanta, Georgia
}

\author{
Charlotte-Paige Rolle ${ }^{1}$, Eli S Rosenberg ${ }^{2}$, Nicole Luisi ${ }^{2}$, Jeremy Grey, Travis Sanchez ${ }^{2}$, \\ Carlos del Rio ${ }^{1,3}$, John L Peterson ${ }^{4}$, Paula M Frew ${ }^{1,3,5}$, Patrick S Sullivan², and Colleen F \\ Kelley ${ }^{1,2}$ \\ ${ }^{1}$ Division of Infectious Diseases, Emory University School of Medicine, Atlanta, GA, USA \\ ${ }^{2}$ Department of Epidemiology, Rollins School of Public Health, Emory University, Atlanta, GA, \\ USA
}

${ }^{3}$ Hubert Department of Global Health, Rollins School of Public Health, Emory University, Atlanta, GA, USA

${ }^{4}$ Department of Psychology, Georgia State University, Atlanta, GA, USA

${ }^{5}$ Department of Behavioral Sciences and Health Education, Rollins School of Public Health, Emory University, Atlanta, GA, USA

\section{Abstract}

PrEP willingness may be different among black and white men who have sex with men (MSM) given known disparities in HIV incidence, sociodemographic factors, and healthcare access between these groups. We surveyed 482 black and white HIV-negative MSM in Atlanta, GA about their willingness to use pre-exposure prophylaxis (PrEP) and facilitators and barriers to PrEP willingness. Overall, 45\% (215/482) of men indicated interest in using PrEP. Engaging in recent unprotected anal intercourse (UAI) was the only factor significantly associated with PrEP willingness in multivariate analyses (OR 1.73, 95\% CI 1.13, 2.65). Willing men identified "extra protection" against HIV as the most common reason for interest in using PrEP, whereas unwilling men most commonly cited not wanting to take medication daily, and this reason was more common among white MSM (42.3\% of white MSM vs. $28.9 \%$ of black MSM, f $>=0.04)$. Most men indicated willingness to use PrEP if cost was $<50$ dollars/month; however, more black MSM indicated willingness to use PrEP only if cost were free (17.9\% of white MSM vs. $25.9 \%$ of black MSM, $p=0.03$ ). Overall, these data are useful to scale up PrEP interventions targeting at-risk MSM in Atlanta and highlight the need for implementation of low cost-programs, which will be especially important for black MSM.

Reprints and permissions: sagepub.co.uk/journalsPermissions.nav

Corresponding author: Charlotte-Paige Rolle, MD MPH, Division of Infectious Disease, Emory University School of Medicine, 1518 Clifton Road NE, Atlanta 30322, GA, USA. crolle@emory.edu.

Declaration of conflicting interests

The authors declared no potential conflicts of interest with respect to the research, authorship, and/or publication of this article. 


\section{Keywords}

Pre-exposure prophylaxis; MSM; willingness; barriers; race; disparities; HIV; prevention

\section{Introduction}

In 2014, Metro Atlanta had the seventh highest number of new HIV diagnoses in the USA; $80 \%$ of these cases occurred among men and $75 \%$ of cases among men were attributed to male-male sexual transmission. Black MSM are disproportionately affected compared to white MSM, and our group previously reported alarming HIV incidence rates of $11 \%$ per year among black MSM aged 18-24 years in Atlanta compared to incidence rates of 1\% among white MSM within the same age group. This racial disparity in HIV infection is seen throughout the USA and persists, despite comparable or lower HIV sexual risk behaviors among black MSM compared to white MSM.- Reductions in racial disparities of HIV infection among MSM in Atlanta and elsewhere may therefore require other effective prevention interventions that do not simply focus on changes in sexual risk behavior. HIV pre exposure prophylaxis (PrEP) has been proposed as a potential solution to address such racial disparities and data to inform scale up of this biomedical intervention are urgently needed in A tlanta, particularly among black MSM.

PrEP with daily Tenofovir/emtricitabine has shown greater than $90 \%$ efficacy in reducing HIV infections among MSM when taken regularly. ${ }^{-}$Cost-effective ness analyses also support targeted use in high risk groups such as MSM, and in 2014, the CDC published clinical practice guidelines for the use of PrEP in high-risk populations. However, PrEP uptake in Atlanta has been slow compared to other US cities; recent data show that only 2$5 \%$ of MSM in Atlanta report using PrEP.' Previous studies have reported that $28-81 \%$ of MSM are interested in using PrEP;- however, no study to date has examined whether motivations to use PrEP are different between black and white MSM. Given the disparities in HIV incidence, sociodemographic factors, and healthcare access among black and white MSM, we hypothesized that factors associated with PrEP willingness may differ between black and white MSM. A detailed understanding of these differences is required to inform targeted PrEP education and uptake efforts. Here, we describe factors associated with PrEP willingness in a large sample of white and black MSM in Atlanta, GA and examine whether these factors differ significantly by race.

\section{Methods}

We conducted a cross-sectional analysis of PrEP interest among MSM using data from the Emory-based InvolveMENt cohort study conducted from 2010 to 2014. A full description of the cohort including recruitment methods, inclusion and exclusion criteria, and primary study outcomes was previously published.' Briefly, 562 HIV-negative black and white MSM aged $\geq 18$ years were recruited from the Atlanta community and Facebook and followed prospectively for up to 24 months. Participants were tested for HIV and other sexually transmitted infections (STIs) including syphilis, urethral and rectal chlamydia and gonorrhea by nucleic acid amplification and completed a computer-assisted self-interview 
questionnaire to assess individual, dyadic, and community level HIV risk factors at the baseline study visit and subsequent study visits every 3-6 months. Questionnaire items were created using Bronfenbrenner'secological systems model to study the individual, social and cultural influences contributing to the disparity in HIV infection between white and black MSM.

From February 2012 to April 2013, participants received a one-time cross-sectional survey querying their interest in using PrEP to prevent HIV infection. The survey was administered at the first scheduled study visit following creation of the PrEP questionnaire items, hence participants received the survey at different follow-up visits depending on their study enrollment date. They were provided with a brief description of PrEP as follows:

Recently, research showed that gay/bisexual men who took a Truvada pill once a day had half the chances of getting HIV compared to men who took a sugar pill. Truvada is a medication that is commonly used to treat HIV infection. To prevent HIV infection, you would need to take this medication every day whether you planned to have sex that day or not. The medication would have to be prescribed by a doctor who would need to see you at least every 3 months to test you for HIV infection. Some insurance companies may not pay for this use of Truvada and you might have to pay for it yourself. There are some side effects reported by people who start taking Truvada, mostly nausea and weight loss that goes away after the first month or so. In rare cases, Truvada taken for long periods can damage the kidneys.

Men were subsequently asked, "Would you be interested in taking Truvada to prevent HIV infection?" and were allowed to answer "Yes, No, or Don't Know".

Men were asked to select reasons for PrEP willingness and unwillingness from a list of options and were allowed to choose all that applied. As PrEP was only recently FDAapproved during the course of this study, data examining motivations for and barriers to use were limited. Therefore, items offered for willingness and unwillingness were created based on expert consultation as well as previous literature regarding willingness to use PrEP (e.g. fear of side effects) and other forms of prophylaxis (e.g. condoms)." Men were also asked how much they would be willing to pay monthly for PrEP. Participant data on demographics, income, insurance status, healthcare access, STI and HIV incidence during study follow-up, behavioral risk, and HIV testing behaviors were obtained from the InvolveMENt database. MSM were defined as having regular healthcare access if they reported having a regular health care provider or receiving care in an on-campus health center. Substance use was defined as the self-reported use of non-injection or injection drugs not prescribed by a provider in the previous 12 months. As a biologic marker of behavioral risk, men who were diagnosed with either an STI or HIV during study follow-up were classified into a single 'Incident HIV/STI' categorical variable for analyses. We defined men as having 'low risk behavior' if they reported either not being sexually active, believing that their partner was HIV-negative, or using condoms $100 \%$ of the time. Informed consent was obtained for all participants, and the Institutional Review Board at Emory University approved study procedures. 
Associations between demographic and risk behavior variables with PrEP willingness were examined with unadjusted univariate logistic regression. The factors chosen for analysis were based on previously published data examining PrEP willingness among diverse samples of MSM and included socioeconomic factors hypothesized to affect PrEP access based on prior reports." Variables with a $p$-value $<0.1$ were included in a stepwise multivariate logistic regression model to detennine which variables remained associated with PrEP willingness after adjustment for potential confounders. The number of unprotected anal sex partners in the last 12 months was not included in the multivariate model due to collinearity with any reported UAI in the last 12 months. We compared reasons for PrEP willingness or unwillingness between black and white MSM with unadjusted Chi square tests. All statistical analyses were conducted using SAS version 9.4.

\section{Results and discussion}

A total of 482 MSM completed the PrEP willingness questions, including 219 (45.4\%) black and 263 (54.6\%) white MSM (see Table 1 for demographic information). Eighty men did not complete the PrEP survey due to HIV seroconversion and/or study completion prior to questionnaire administration. The median age of the sample was 26 years (IQR 22, 31). Most had at least some college education $(84 \%, 406 / 482)$ and health insurance coverage $(67 \%, 321 / 482)$. The median number of sexual partners in the six months prior to study enrollment was 3 (IQR 2-3), and 71\% (344/482) reported unprotected anal intercourse (UAI) with at least one partner in the previous 12 months. Of MSM who completed the PrEP questions, 138/482 (28.6\%) were diagnosed with an STI and 20/482 (4.1\%) were diagnosed with HIV during study follow-up. Compared to white MSM, black MSM in the InvolveMENt cohort were younger, less educated, had lower income, were less likely to have health insurance or regular healthcare access, and were more likely to be diagnosed with an incident STI or HIV during study follow-up as was previously reported.' White MSM were more likely to report UAI and were more likely to have a greater number of sexual partners.

Data summarizing factors associated with PrEP willingness are presented in Table 2. Overall, 215/482 men (45\%) reported that they would be willing to use PrEP including 98/219 (44.7\%) black MSM and 117/263 (44.5\%) white MSM (OR 1.01, 95\% CI 0.7-1.45). Univariate analyses demonstrated that any reported UAI in the last 12 months (OR 1.73, 95\% CI 1.14-2.61) and reported UAI with 3+ partners in the last 12 months (OR 2.34, 95\% CI 1.40-3.91) were significantly associated with PrEP willingness. Being uninsured (OR 1.43, 95\% CI 0.97-2.11) and being diagnosed with incident HIV/STI during study follow-up (OR 1.46, 95\% CI 0.99-2.15) were positively associated with PrEP willingness but did not reach statistical significance. Race, age, education, income, having regular healthcare access, recent homelessness, and substance use were not significantly associated with PrEP willingness. When stratifying by race, there was also no association between age, education, income, having regular healthcare access, recent homelessness, and substance use and PrEP willingness. In multivariable analysis, reported UAI in the last 12 months was the only variable significantly associated with PrEP willingness (OR 1.73, 95\% CI 1.13-2.65). 
The most common reasons reported for PrEP willingness included interest in additional HIV protection options [88\% (103/117) of white MSM vs. 73\% (72/98) of black MSM, $p=0.2]$ and doctor recommendation to use PrEP, although black MSM were less likely to endorse a doctor's recommendation for PrEP willingness compared to white MSM [45\% (53/117) of white MSM vs. $22 \%$ (22/98) of black MSM, $p=0.002$ ] (Table 3). Nonetheless, 24\% (18/75) of men who endorsed recommendation from a doctor as a reason for PrEP willingness were uninsured and $38.7 \%$ (29/75) did not have regular access to healthcare. The most common reasons reported for PrEP unwillingness included not wanting to take a daily medication [42.3\% (62/146) of white MSM vs. 28.9\% (35/121) of black MSM, $p=0.04]$ and not being able to afford the medication [35.6\% (52/146) of white MSM vs.28.1\% (34/121) of black MSM, $p=0.2$ ] (Table 4). White MSM were more likely than black MSM to endorse not wanting to take daily medication [42.3\% (62/146) vs. $28.9 \%(35 / 121), p=0.04]$, not wanting to see a doctor regularly [24.7\% (36/146) vs. $7.4 \%(9 / 121),=0.0003]$, and belief in a partner's negative HIV status [32.9\% (48/146) vs. $10.7 \%$ (13/121), $p \leq 0.0001]$ as reasons for being unwilling to take PrEP. In comparison, black MSM were more likely than white MSM to cite not being sexually active as a reason for PrEP unwillingness [17.4\% (21/121) vs. $7.5 \%(11 / 146), p=0.02]$. Of men who chose reasons defined as "low risk behavior" for PrEP unwillingness, 56.2\% (77/137) reported UAI in the last 12 months and 28.5\% (39/137) were diagnosed with an STI or HIV during the study follow-up period.

White MSM were willing to pay more per month for PrEP, 61.5\% (158/257) of white MSM were willing to pay up to $\$ 50$ dollars per month compared to only $47.2 \%(102 / 216)$ of black $\operatorname{MSM}(p=0.003)$. In addition, more black MSM indicated willingness to use PrEP only if it were free [17.9\% (46/257) of white MSM vs. 25.9\% (56/216) of black MSM, $p=0.03$ ].

Approximately half of black and white MSM in Atlanta reported willingness to use PrEP, which is slightly lower than previously published estimates from other Atlanta-based samples of black MSM which report that $60-81 \%$ of men are willing to use PrEP.' Possible reasons for this discrepancy include differences in sampling methodology; we utilized venue-based sampling to recruit men whereas one of the prior studies recruited men attending a large Black Gay pride event, hence respondents who attended this community event may be different from black MSM recruited at other venues. Second, it is not known what information about PrEP was provided to participants in these other studies and differences in descriptions of PrEP (i.e. regarding efficacy and side effects) may also partly explain the higher willingness estimates observed in other Atlanta-based samples. Our data are novel in that this is the first study to compare PrEP willingness between black and white MSM in the southeastern USA and findings are largely consistent with willingness studies involving MSM from other geographic regions.-- Additional data examining factors surrounding PrEP use in the Southern US are urgently needed as this area continues to experience the highest burden of HIV infection in the USA and PrEP has the potential to significantly reduce both geographical and racial disparities in HIV infection if targeted towards populations at highest risk.

The only factor significantly associated in multivariable analysis with PrEP willingness was engaging in UAI. This association of PrEP willingness with high-risk behavior has been previously demonstrated ${ }^{-}$and is encouraging given $\mathrm{CDC}$ recommendations for targeting 
PrEP use in high-risk groups. We originally hypothesized that race, income, education and lack of insurance might be associated with PrEP willingness due to perceived barriers in accessing this expensive biomedical intervention. While our study may have lacked sufficient power, the lack of association between PrEP willingness and these social determinants of health is salient when considering PrEP implementation for black MSM in Atlanta, many of whom are uninsured, live in poverty, and live in a state that has not expanded Medicaid.' It is imperative that PrEP delivery programs serving black MSM offer solutions to ensure that education, cost, and healthcare access do not serve as barriers for men who want and are in need of PrEP.

In our analysis, race was not associated with willingness to use PrEP; however, black and white MSM did endorse different motivations and barriers to PrEP use. White MSM were more likely to report PrEP interest if a doctor recommended it. This may be reflective of better access to healthcare among white MSM and/or evidence of medical mistrust among black MSM who may be less confident in provider recommendations based on their racial and sexual identity perceptions about medical culture and healthcare. Race-based medical mistrust can serve as an important deterrent to accessing healthcare and preventive services and has previously been shown to be a significant factor adversely affecting willingness to use PrEP among black MSM. These findings suggest that optimizing PrEP delivery to black MSM will require strategies to improve relationships with healthcare providers and ensure culturally sensitive PrEP education to address concerns related to PrEP use.

White MSM who reported lack of PrEP interest were more likely than black MSM to endorse not wanting to take a medication daily and not wanting to go to a doctor regularly for follow-up. This is consistent with prior studies that have highlighted daily adherence and frequent provider visits as significant barriers to PrEP use, especially for white MSM.' Current daily dosing and monitoring recommendations for PrEP may be burdensome to patients and providers. Our data further support the development of innovative PrEP delivery programs and long-acting PrEP options for MSM who find daily dosing and frequent provider visits to be a deterrent to PrEP uptake.

Many MSM in our study chose reasons we defined as 'low risk behavior' for PrEP unwillingness, including not being sexually active, believing that their partner was HIVnegative, or using condoms $100 \%$ of the time. However, $56 \%$ of those with low risk behavior reported UAI in the previous 12 months, and $28.5 \%$ were diagnosed with HIV or an STI during study follow-up demonstrating some degree of discordance between perceived and actual risk. Findings from the US PrEP Demonstration Project also noted a similar discrepancy between perceived and actual HIV risk. Formal population-specific risk assessment tools which combine risk-behavior assessment with local epidemiologic risk are urgently needed to identify and maximize PrEP uptake in high-risk MSM who would most benefit from PrEP.

Cost of PrEP was an important barrier identified by many black MSM in our study with 26\% only willing to use PrEP if it was free. Studies have shown prescription costs to be a significant barrier associated with PrEP use"; however, this may be moderated by the manufacturer's assistance program which provides robust co-pay assistance and free drug to 
uninsured low-income men. Despite drug coverage, costs associated with provider visits and lab monitoring may still render PrEP inaccessible for uninsured and low income men due to co-pays and out-of-pocket costs. We have previously demonstrated how structural barriers (i.e. lack of health insurance and healthcare access) may theoretically limit PrEP uptake for Atlanta MSM; however, the current analysis reveals that these structural barriers do not seem to affect willingness to use PrEP. Nonetheless, our data further highlight the need for open access, low cost services to ensure that potential disparities in PrEP uptake do not further exacerbate the observed racial disparities in HIV infection.

This study is limited by its cross-sectional design and lack of follow-up to determine actual PrEP uptake in our cohort. The PrEP questionnaire was completed by $81.5 \%$ (393/482) of men just prior to FDA approval of PrEP and willingness to use PrEP could be significantly different given recent dramatic increases in PrEP knowledge and uptake.' Lastly, our sample focuses on MSM from a single city in the southeastern USA and is not generalizable to all MSM.

\section{Conclusions}

Our findings highlight important reasons for willingness to use PrEP, and to some extent, these reasons differ between white and black MSM. Prospective studies examining barriers and facilitators to PrEP uptake among black and white MSM are needed to clarify these differences as PrEP offers significant potential to reduce HIV incidence and disparities among black and white MSM.

\section{Acknowledgements}

We would like to acknowledge Dr Dawn Smith from the Centers for Disease Control and Prevention (CDC) who helped create the InvolveMENt PrEP questionnaire. We would also like to acknowledge the Involvement study participants and dedicated study staff who contributed immensely to this work.

Funding

The authors disclosed receipt of the following financial support for the research, authorship, and/or publication of this article: This work was supported by the National Institutes of Health: KL2TR000455 (PI: Kelley), K23AI108335 (PI: Kelley), R01MH085600 (PI: Sullivan), R01DA038196-01 (PI: Sullivan/Rosenberg), The Atlanta Clinical and Translational Science Institute UL1TR000454, and the Emory Center for AIDS Research P30 AI050409.

\section{References}

1. Centers for Disease Control and Prevention. HIV surveillance report, 2014, vol. 26 Available at: http://www.cdc.gov/hiv/library/reports/surveillance/ (2015, accessed 30 December 2015).

2. Georgia Department of Public Health, HIV/AIDS Epidemiology Section. HIV surveillance summary, Georgia, 2014. Available at: https://dph.georgia.gov/data-fact-sheet-shummaris (2016, accessed 15 April 2016).

3. Sullivan PS, Rosenberg ES, Sanchez TH, et al. Explaining racial disparities in HIV incidence in black and white men who have sex with men in Atlanta, GA: a prospective observational cohort study. Ann Epidemiol 2015; 25: 445-154. [PubMed: 25911980]

4. Feldman MB. A critical literature review to identify possible causes of higher rates of HIV infection among young black and Latino men who have sex with men. J Natl Med Assoc 2010; 102: 12061221. [PubMed: 21287902] 
5. Millett GA, Flores SA, Peterson JL, et al. Explaining dis parities in HIV infection among black and white men who have sex with men: a meta-analysis of HIV risk behaviors. AIDS 2007; 21: 20832091. [PubMed: 17885299]

6. Millett GA, Peterson JL, Flores SA, et al. Comparisons of disparities and risks of HIV infection in black and other men who have sex with men in Canada, UK, and USA: a meta-analysis. Lancet 2012; 380: 341-348. [PubMed: 22819656]

7. Office of National AIDS Policy National HIV/AIDS Strategy for the United States: Updated to 2020, https://www.aids.gov/federal-resources/national-hiv-aids-strategy/nhas-update.pdf (2015, accessed 22 August 2016).

8. Grant RM, Lama JR, Anderson PL, et al. Preexposure chemoprophylaxis for HIV prevention in men who have sex with men. New Engl J Med 2010; 363: 2587-2599. [PubMed: 21091279]

9. United States Public Health Service. Pre-exposure prophylaxis for the prevention of HIV infection in the United States - 2014, a clinical practice guideline, http://www.cdc.gov/hiv/pdf/ prepguidelines2014.pdf (2014, accessed 12 December 2015).

10. Liu AY, Cohen SE, Yittinghoff E, et al. Preexposure prophylaxis for hiv infection integrated with municipal and community-based sexual health services. JAMA Intern Med 2016; 176: 75-84. [PubMed: 26571482]

11. Volk JE, Marcus JL, Phengrasamy T, et al. No new HIV infections with increasing use of HIV preexposure prophylaxis in a clinical practice setting. Clin Infect Dis: an official publication of the Infectious Diseases Society of America 2015; 61: 1601-1603.

12. Juusola JL, Brandeau ML, Owens DK, et al. The cost effectiveness of preexposure prophylaxis for HIV prevention in the United States in men who have sex with men. Ann Intern Med 2012; 156: 541-550. [PubMed: 22508731]

13. Delaney KP, Sanchez T, Bowles K, et al. Awareness and use of PrEP appear to be increasing among internet samples of US MSM In: Conferences on retroviruses and opportunistic infections (CROI), Boston, MA, 22-25 22016.

14. Hoots BE, Finlayson T, Nerlander L, et al. Willingness to take, use of, and indications for preexposure prophylaxis among men who have sex with men - 20 US Cities, 2014. Clin Infect Dis 2016; 63: 672-677. [PubMed: 27282710]

15. Cohen SE, Yittinghoff E, Bacon O, et al. High interest in preexposure prophylaxis among men who have sex with men at risk for HIV infection: baseline data from the US PrEP demonstration project. J Acquir Immune Defic Syndr 2015; 68: 439-448. [PubMed: 25501614]

16. Eaton LA, Driffin DD, Smith H, et al. Psychosocial factors related to willingness to use preexposure prophylaxis for HIY prevention among Black men who have sex with men attending a community event. Sex Health 2014; 11: 244-251. [PubMed: 25001553]

17. Grov C, Whitfield TH, Rendina HJ, et al. Willingness to take PrEP and potential for risk compensation among highly sexually active gay and bisexual men. AIDS Behav 2015; 19: 22342244. [PubMed: 25735243]

18. Holt M, Murphy DA, Callander D, et al. Willingness to use HIY pre-exposure prophylaxis and the likelihood of decreased condom use are both associated with unprotected anal intercourse and the perceived likelihood of becoming HIV positive among Australian gay and bisexual men. Sex Transm Infect 2012; 88: 258-263. [PubMed: 22290327]

19. Krakower DS, Mimiaga MJ, Rosenberger JG, et al. Limited awareness and low immediate uptake of preexposure prophylaxis among men who have sex with men using an Internet social networking site. PLoS One 2012; 7: e33119. [PubMed: 22470438]

20. Eaton LA, Driffin DD, Bauermeister J, et al. Minimal awareness and stalled uptake of pre-exposure prophylaxis (PrEP) among at-risk, HIV-negative, black men who have sex with men. AIDS Patient Care STDS 2015; 29: 423-429. [PubMed: 26083143]

21. Sullivan PS, Peterson J, Rosenberg ES, et al. Understanding racial HIV/STI disparities in black and white men who have sex with men: a multilevel approach. PloS One 2014; 9: e90514. [PubMed: 24608176]

22. Bronfenbrenner U and Ceci SJ. Nature-nurture reconceptualized in developmental perspective: a bioecological model. Psychol Rev 1994; 101: 568-586. [PubMed: 7984707] 
23. Smith DK, Toledo L, Smith DJ, et al. Attitudes and program preferences of African-American urban young adults about pre-exposure prophylaxis (PrEP). AIDS Educ Prev 2012; 24: 408-421. [PubMed: 23016502]

24. Malebranche DJ, Peterson JL, Fullilove RE, et al. Race and sexual identity: perceptions about medical culture and healthcare among Black men who have sex with men. J Natl Med Assoc 2004; 96: 97-107. [PubMed: 14746359]

25. Perez-Figueroa RE, Kapadia F, Barton SC, et al. Acceptability of PrEP uptake among racially/ ethnically diverse young men who have sex with men: the P I8 study. AIDS Educ Prevent: official publication of the International Society for AIDS Education 2015; 27: 112-125.

26. Young I and McDaid L. How acceptable are antiretrovirals for the prevention of sexually transmitted HIV?: A review of research on the acceptability of oral pre-expo sure prophylaxis and treatment as prevention. AIDS Behav 2014; 18: 195-216. [PubMed: 23897125]

27. Andrews CD and Heneine W. Cabotegravir long-acting for HIV-1 prevention. Curr Opin HIV AIDS 2015; 10: 258-263. [PubMed: 26049951]

28. Kelley CF, Kahle E, Siegler A, et al. Applying a PrEP continuum of care for men who have sex with men in Atlanta, Georgia. Clin Infect Dis 2015; 61: 1590-1597. [PubMed: 26270691] 







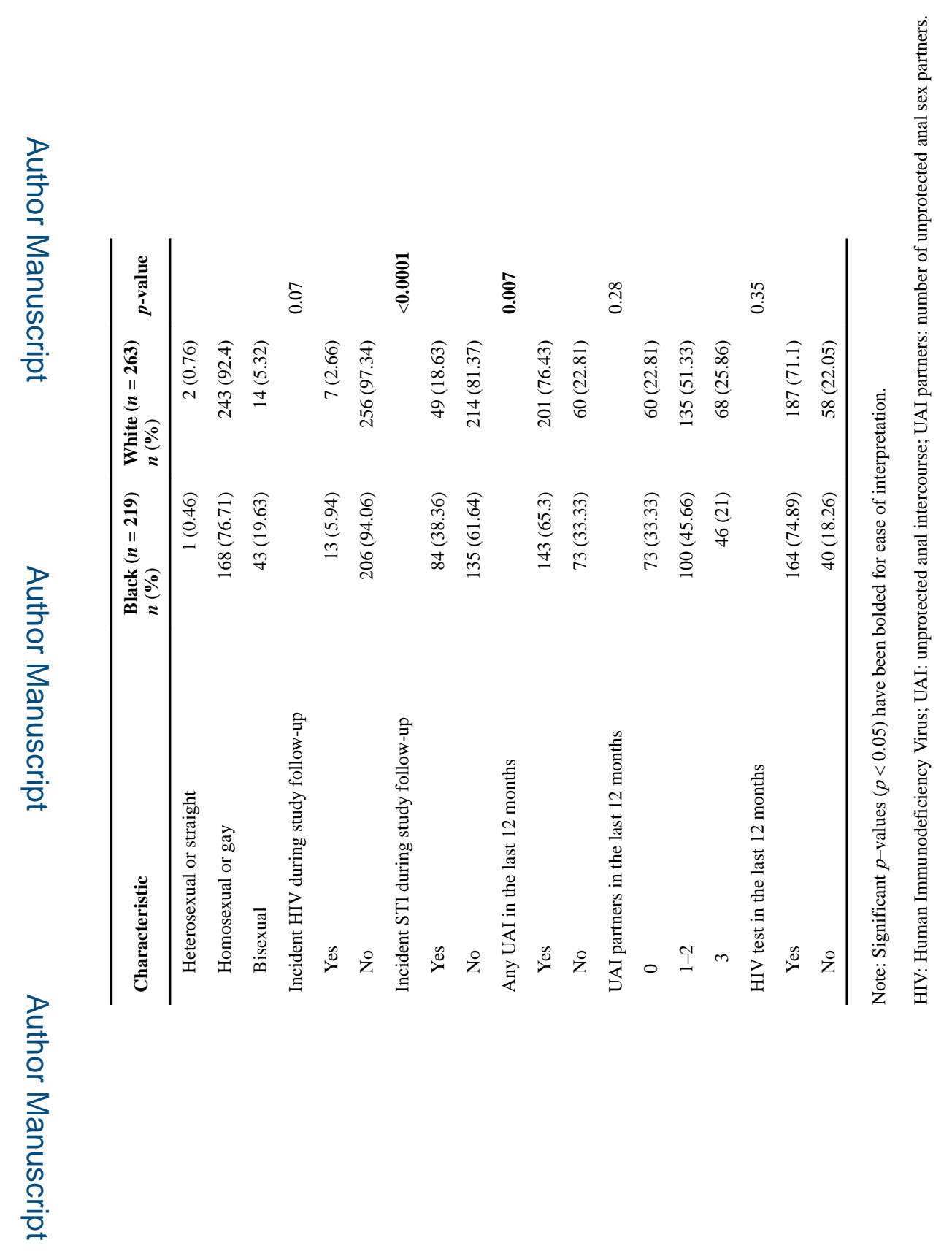

롤

Int J STD AIDS. Author manuscript; available in PMC 2018 October 10. 


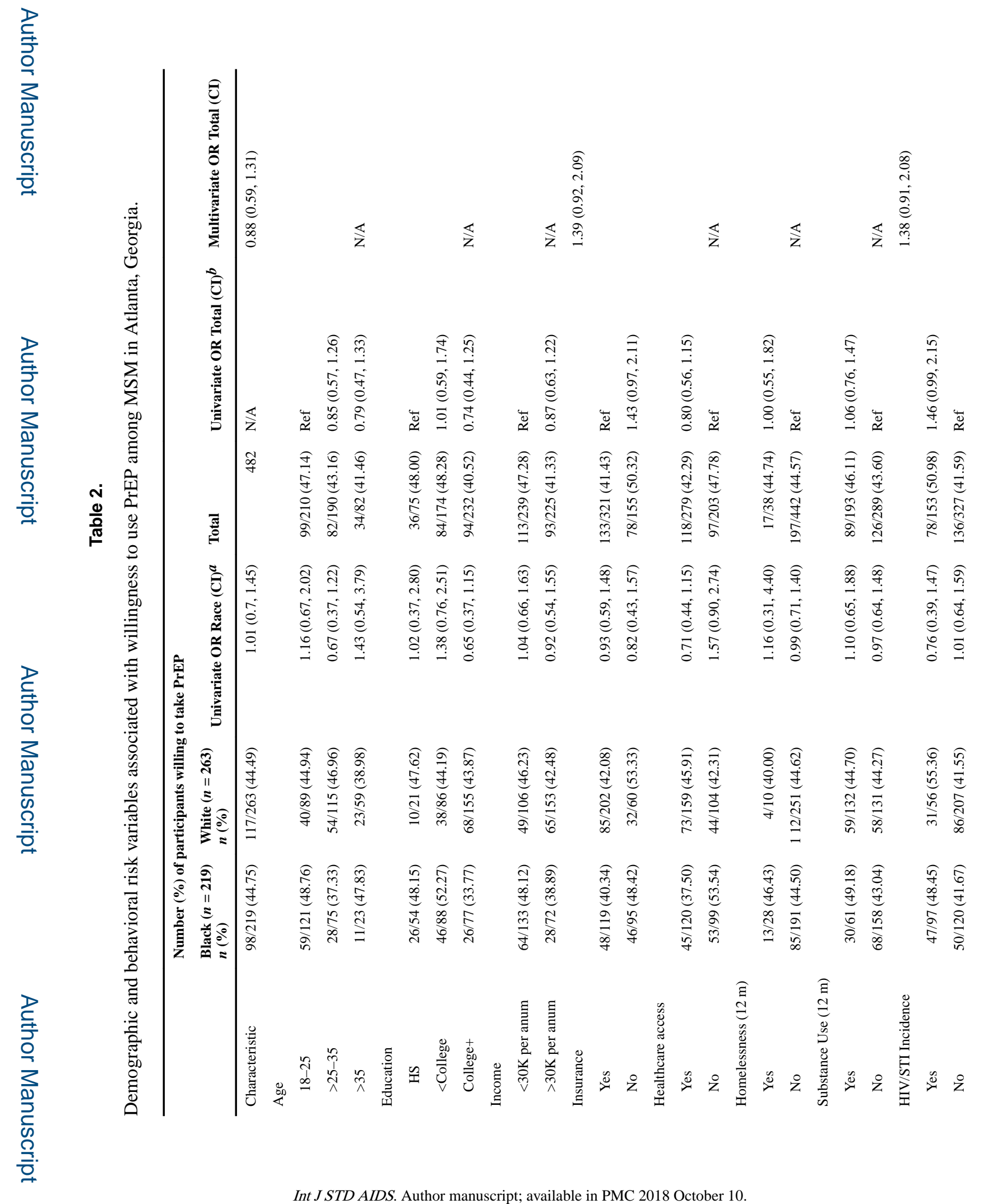




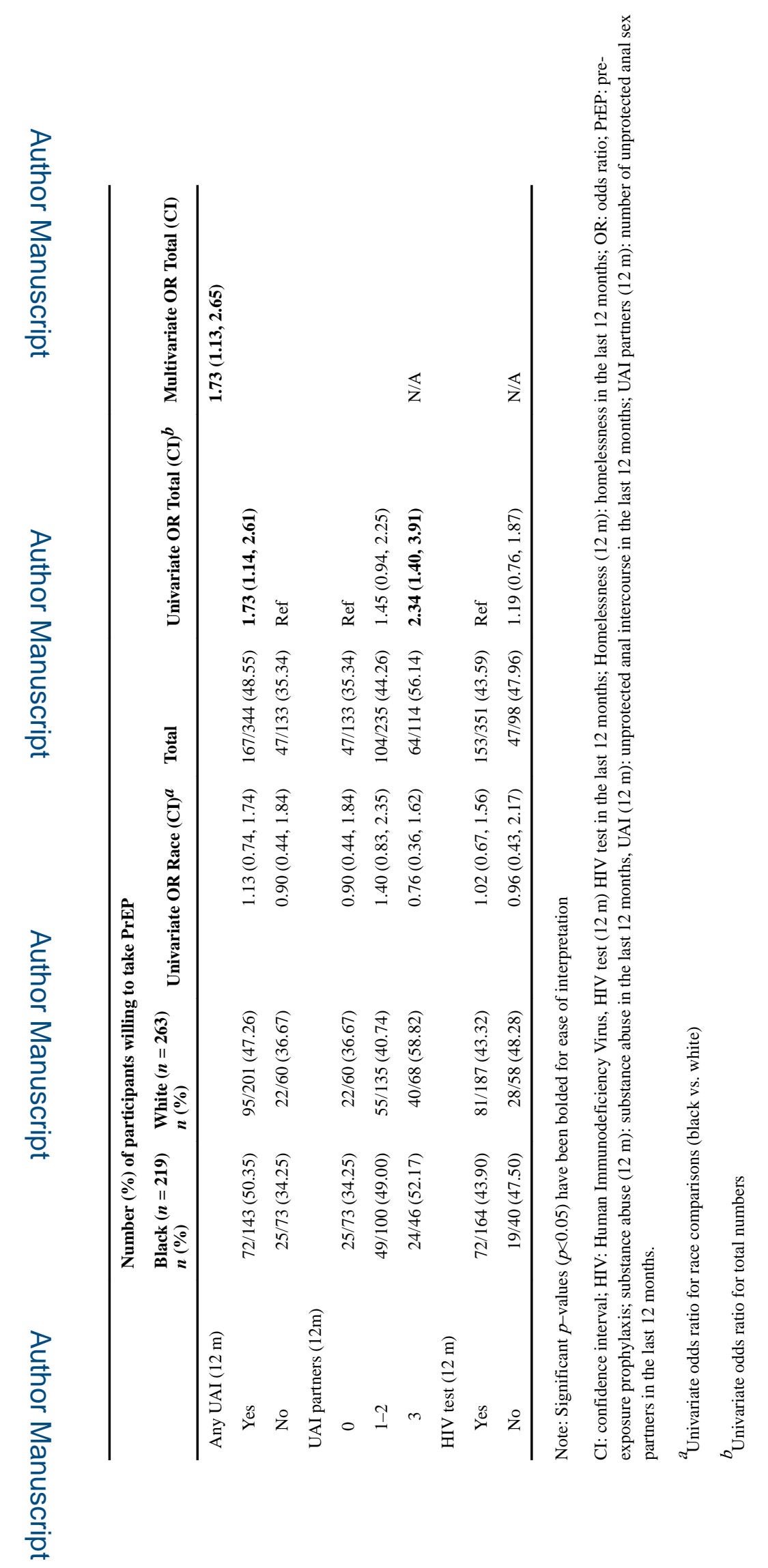

Int J STD AIDS. Author manuscript; available in PMC 2018 October 10. 


\section{롤 \\ 골}

롤

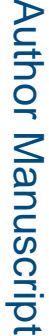

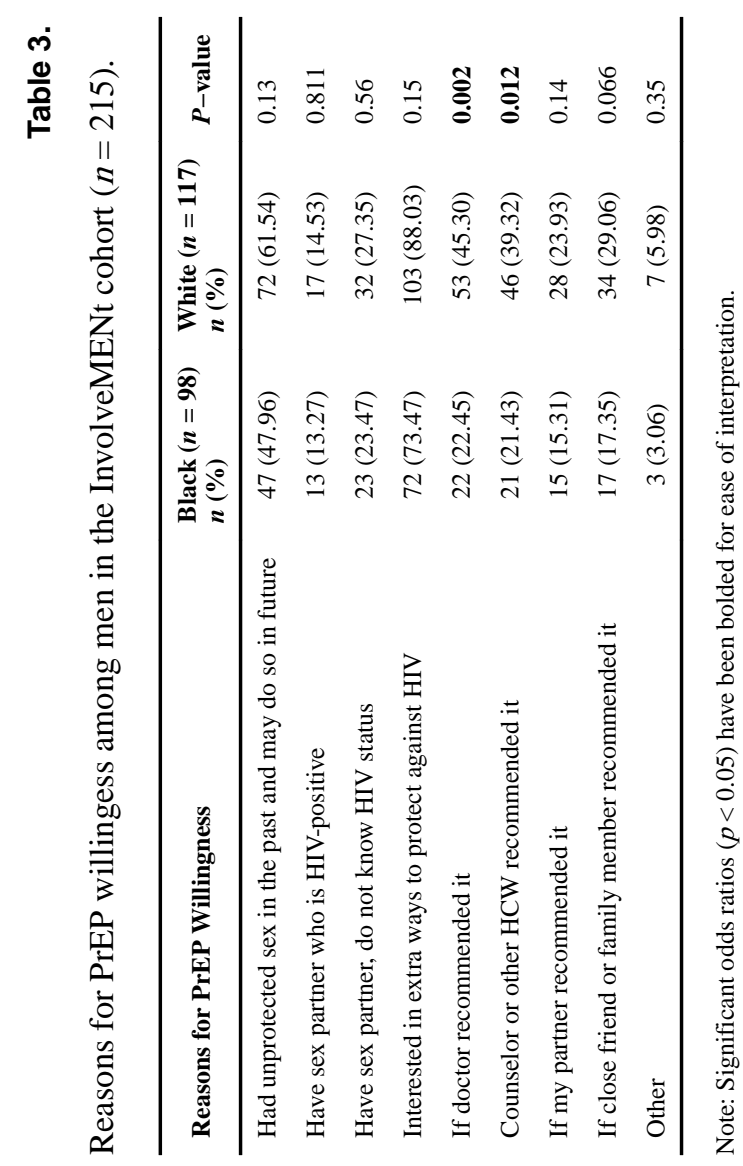

Int J STD AIDS. Author manuscript; available in PMC 2018 October 10. 


\section{롤 \\ 굴}

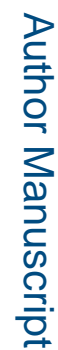

西

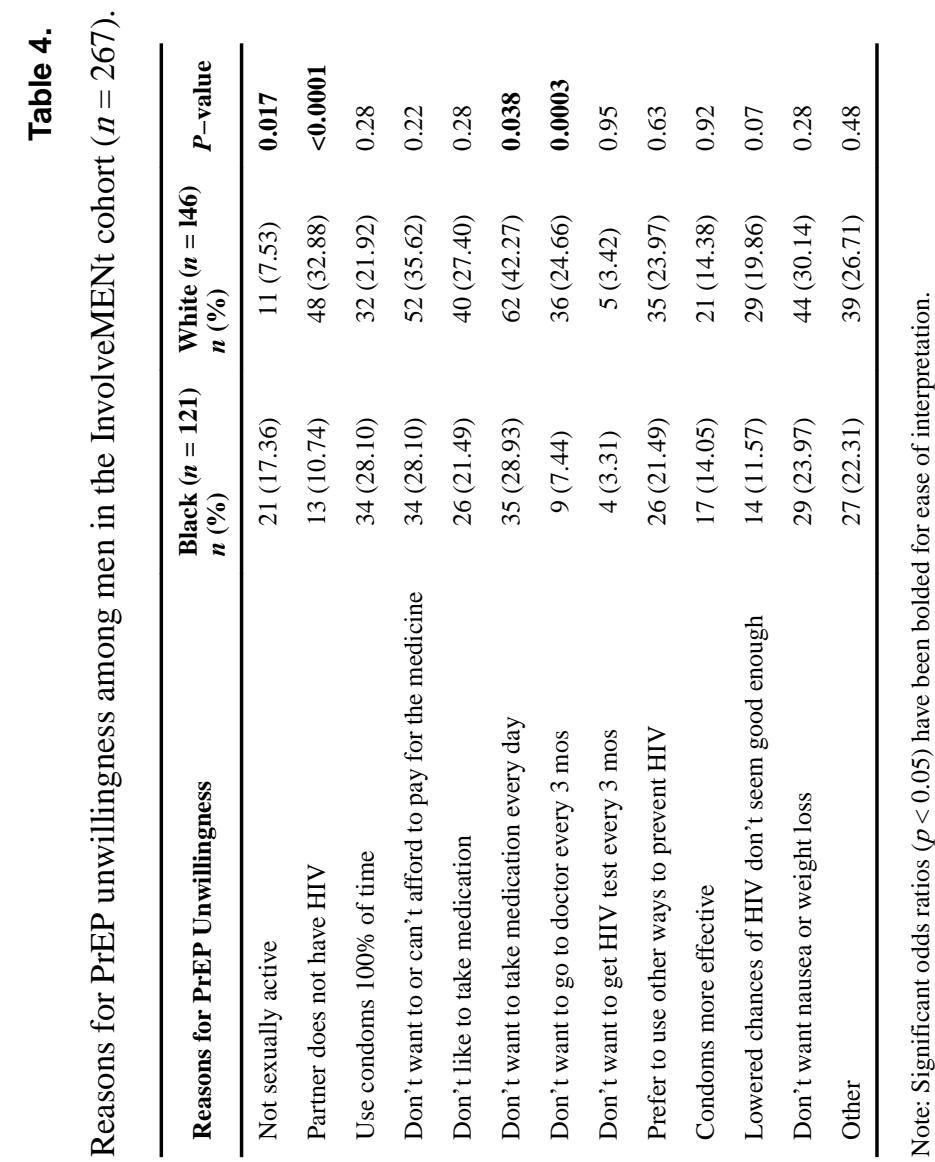

Int J STD AIDS. Author manuscript; available in PMC 2018 October 10. 